\title{
Excellent methanol to olefin performance of SAPO-34 crystal deriving from the mixed micropore, mesopore and macropore architecture
}

Ying-Liang Zhu ${ }^{a}$, Haowen Dai ${ }^{a, b}$, Ying Duan ${ }^{a}$, Qiang Chen ${ }^{*}$, , Meng Zhang ${ }^{*}, a$

a, School of Chemical Engineering and Technology, Sun Yat-sen University, Zhuhai 519082, Guangdong, PR China

b, Hubei Provincial Key Laboratory of Green Materials for Light Industry, Hubei University of Technology, Wuhan 430068, Hubei, PR China

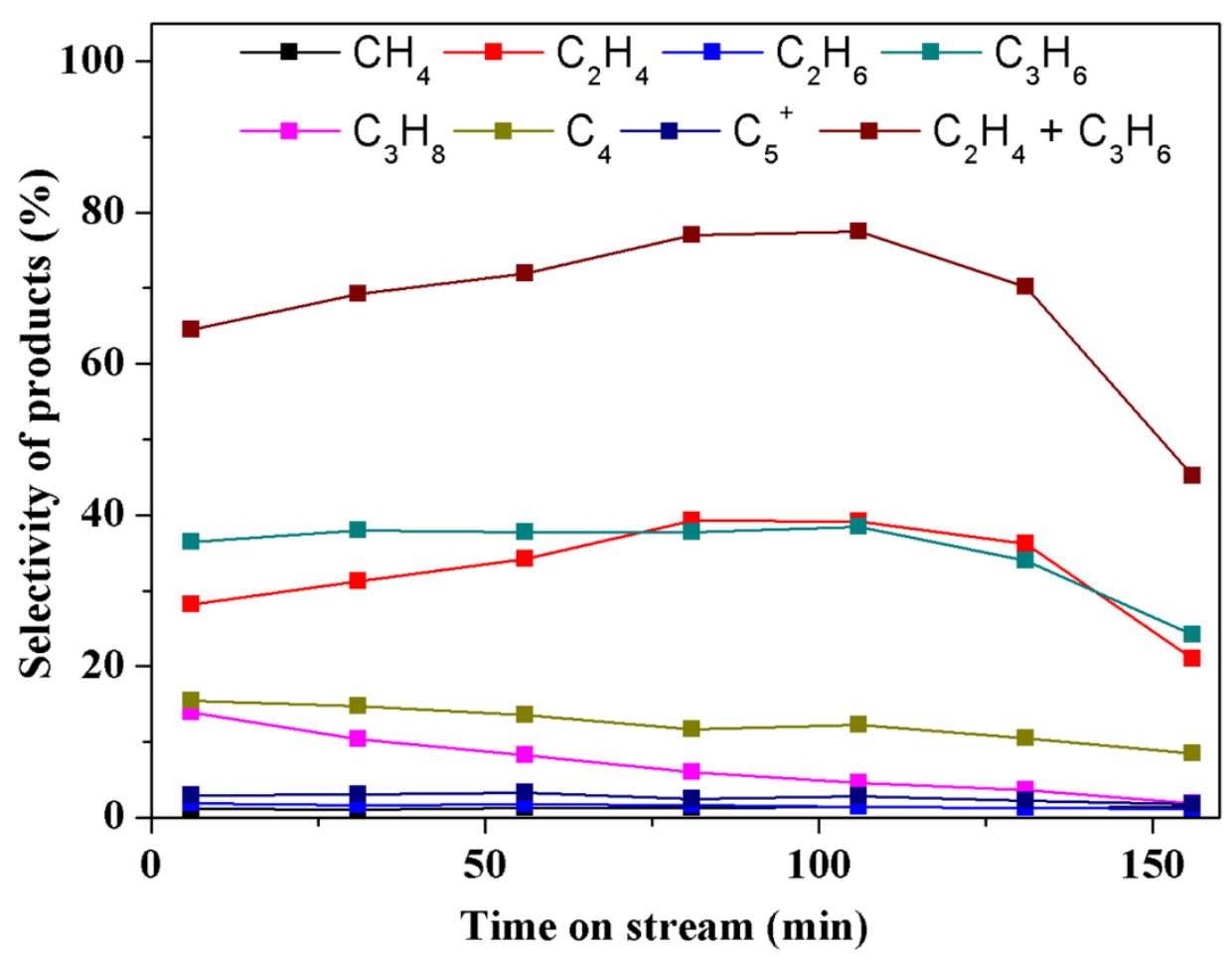

Fig. S1. Selectivity of hydrocarbons over S-0 sample. 


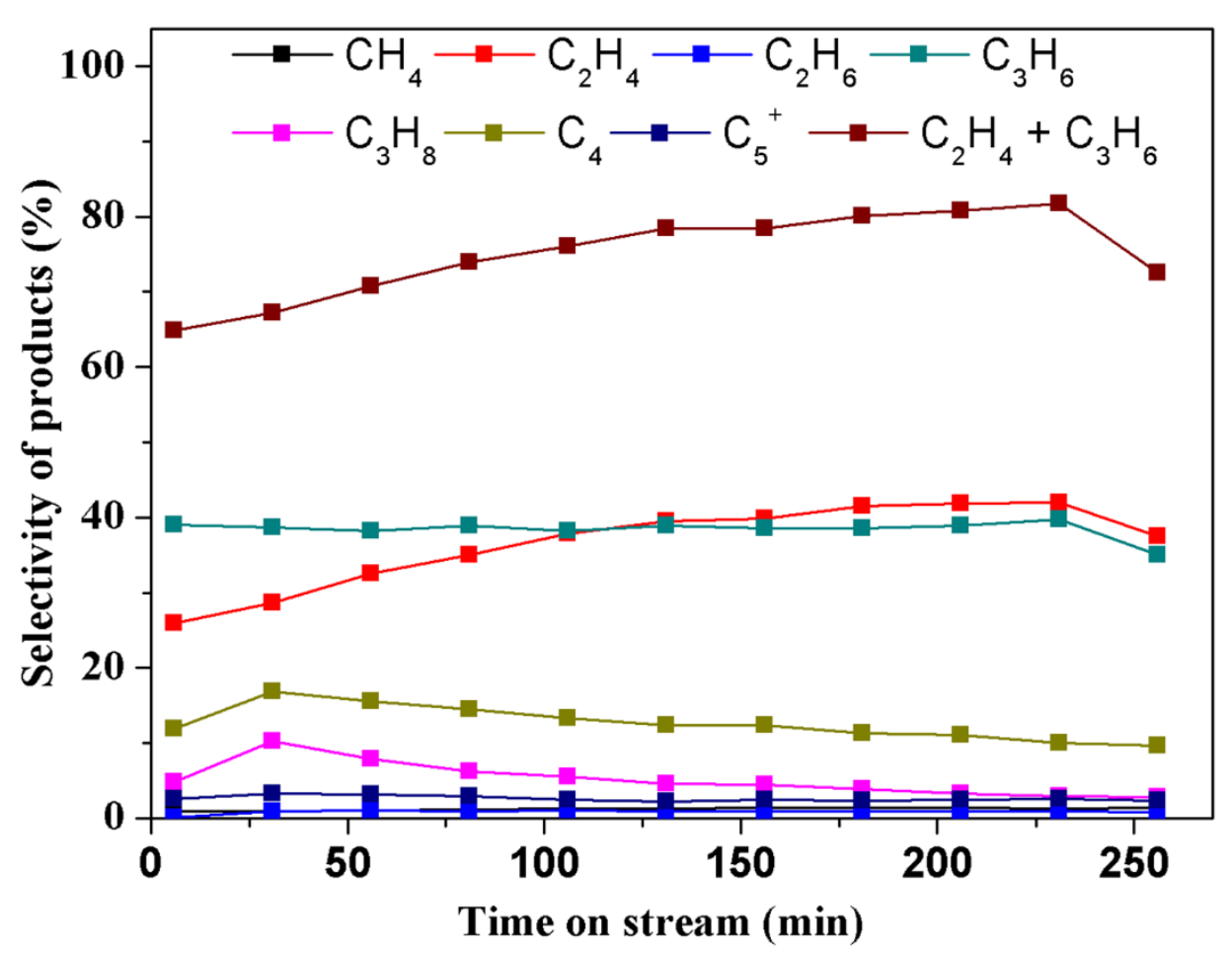

Fig. S2. Selectivity of hydrocarbons over S-24 sample.

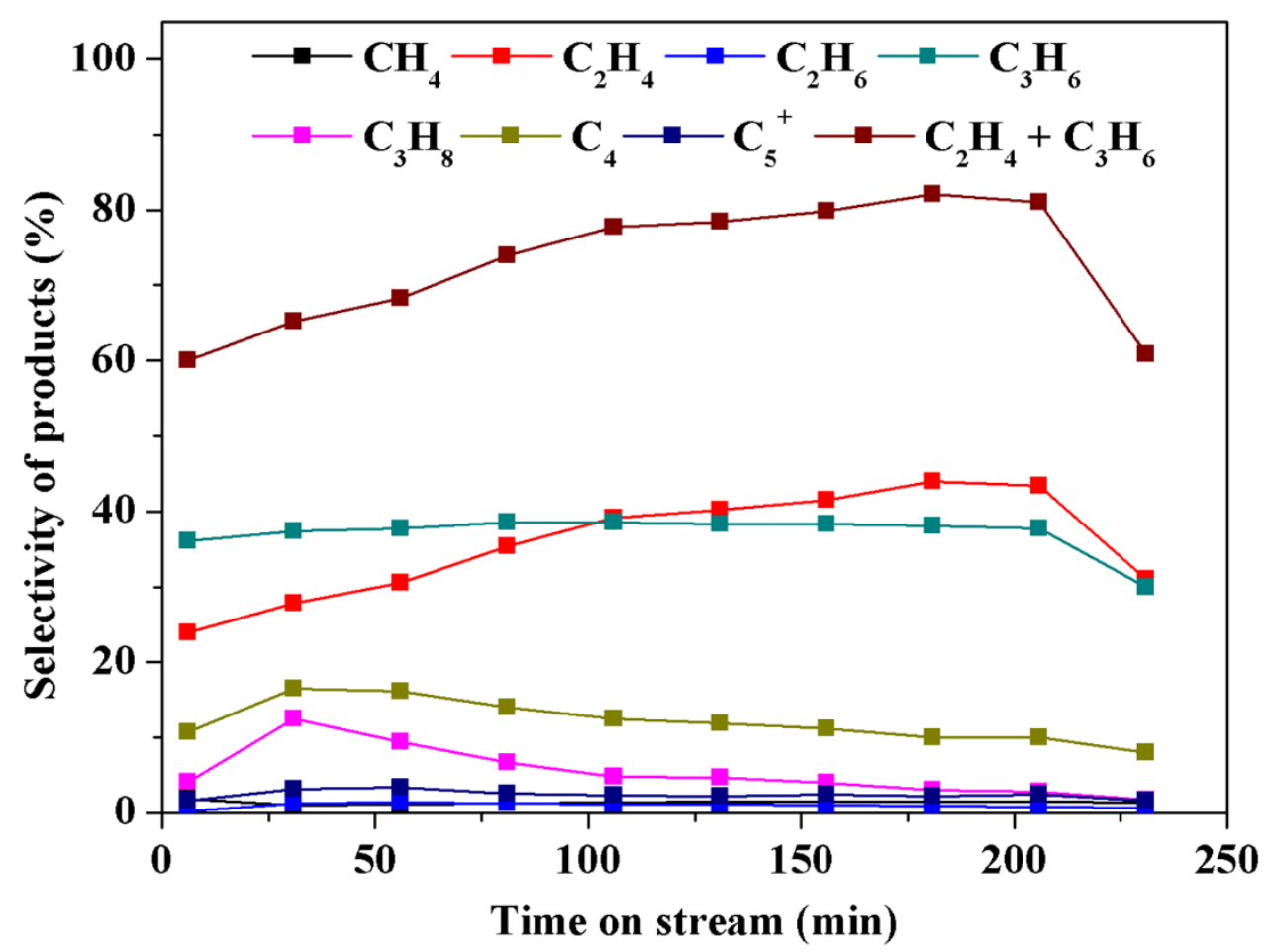

Fig. S3. Selectivity of hydrocarbons over S-48 sample. 


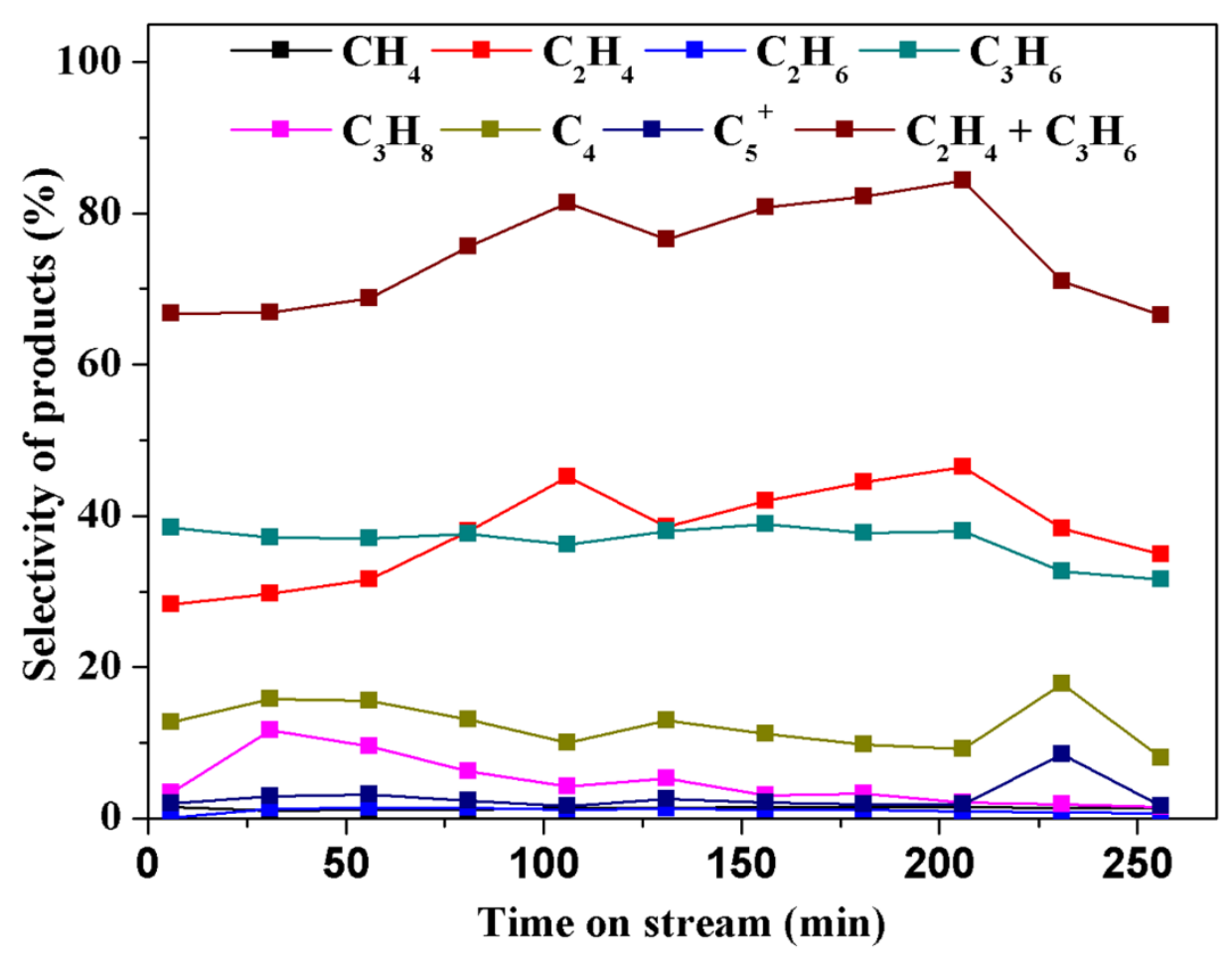

Fig. S4. Selectivity of hydrocarbons over S-72 sample.

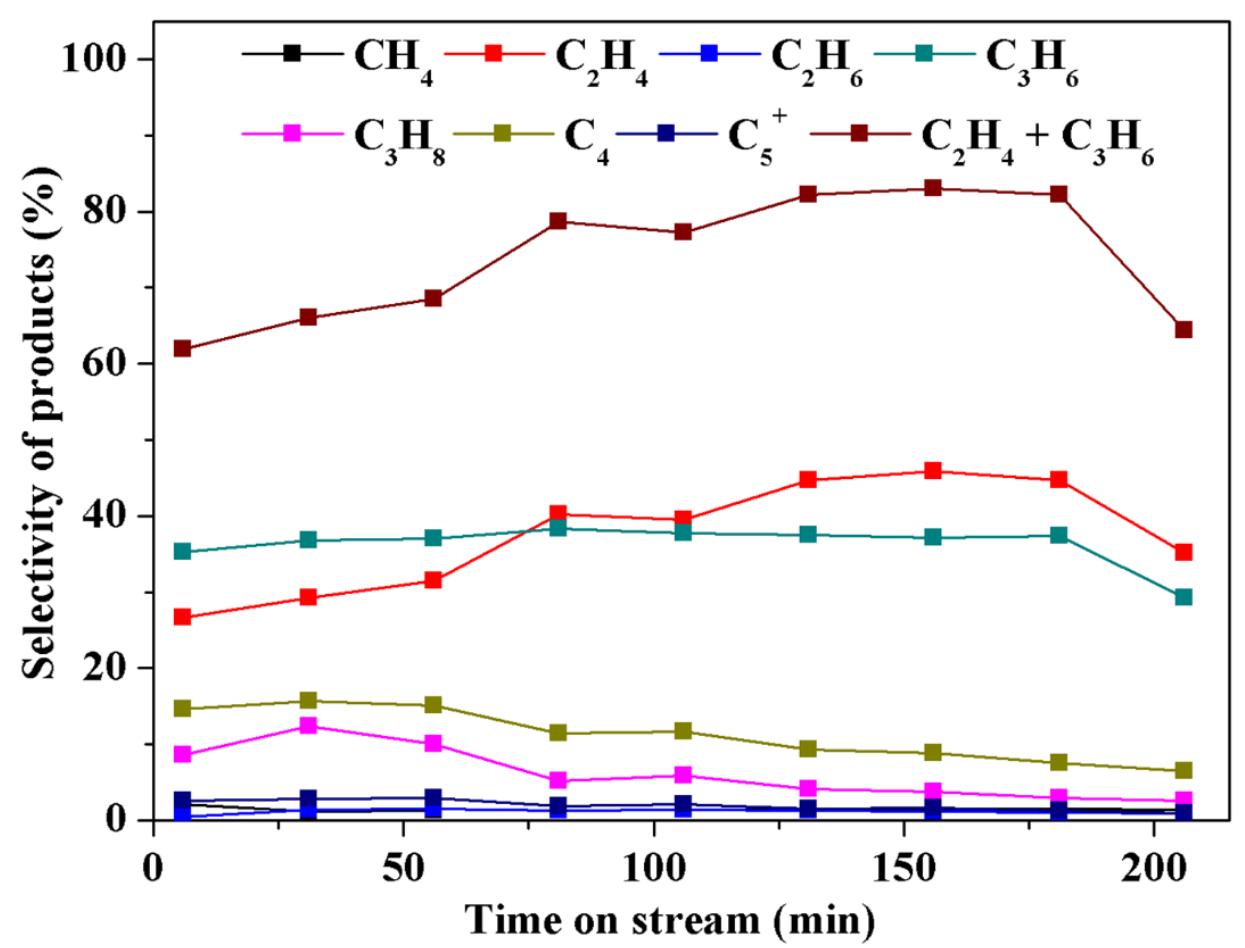

Fig. S5. Selectivity of hydrocarbons over S-120 sample. 
Table S1. Selectivity of hydrocarbons over all samples.

\begin{tabular}{lccccccccc}
\hline \multirow{2}{*}{ Sample } & \multirow{2}{*}{$\begin{array}{c}\text { TOS } \\
(\min )\end{array}$} & $\mathrm{CH}_{4}$ & $\mathrm{C}_{2} \mathrm{H}_{4}$ & $\mathrm{C}_{2} \mathrm{H}_{6}$ & $\mathrm{C}_{3} \mathrm{H}_{6}$ & $\mathrm{C}_{3} \mathrm{H}_{8}$ & $\mathrm{C}_{4}$ & $\mathrm{C}_{5}{ }^{+}$ & $\mathrm{C}_{2}=+\mathrm{C}_{3}{ }^{=}$ \\
\hline $\mathrm{S}-0$ & 106 & 1.369 & 39.13 & 1.444 & 38.40 & 4.557 & 12.23 & 2.666 & 77.53 \\
$\mathrm{~S}-24$ & 231 & 1.305 & 42.01 & 0.881 & 39.69 & 2.898 & 10.04 & 2.579 & 81.70 \\
$\mathrm{~S}-48$ & 206 & 1.488 & 43.95 & 0.973 & 38.13 & 3.097 & 10.02 & 2.208 & 82.08 \\
$\mathrm{~S}-72$ & 206 & 1.471 & 46.41 & 0.887 & 37.94 & 2.054 & 9.237 & 1.904 & 84.35 \\
$\mathrm{~S}-120$ & 181 & 1.443 & 45.87 & 1.181 & 37.16 & 3.813 & 8.851 & 1.578 & 83.03 \\
\hline
\end{tabular}

Experimental condition: $50 \% \mathrm{Wt}$ methanol, WHSV $=2 \mathrm{~h}^{-1}, \mathrm{~T}=450{ }^{\circ} \mathrm{C}$, catalyst weight $=0.5 \mathrm{~g} ;{ }^{*}$ Lifetime: the reaction duration with the conversion of the methanol $\geqq 99 \%$. 